\title{
Research on Key Technologies of Network Security Multidimensional Dynamic Risk Assessment
}

\author{
Jiang Ming', Li Ming ${ }^{1}$, Cai Mengchen², Fang Yuan ${ }^{3}$ \\ ${ }^{1}$ Information and communication branch of State Grid Anhui electric power co., LTD. Hefei, Anhui province \\ 230000 \\ ${ }^{2}$ State Grid Anhui electric power co., LTD. Hefei, Anhui province 230000 \\ ${ }^{3}$ State Grid Anhui power communication co. Hefei, Anhui province 230000
}

Keywords: Security risk, network security, risk assessment, key technology

\begin{abstract}
The emergence of the Internet has had a huge impact on people's lives and work. In the 21st century, human society has fully entered the Internet age. Through the Internet, people can realize zero-distance, data transmission and information sharing without time and space restrictions. The network has become an important tool for modern people to obtain information, disseminate and share information, and has been applied to almost all fields, from scientific research to astronomy, national defense, medical care, small to home entertainment, production office, web browsing must use the network. However, network security issues have always restricted the application of the network. The network has the characteristics of openness, complexity and speciality. Some lawless elements will steal other people's information and finance through cyber attacks. The network security problem cannot be ignored. It is important to do a multi-dimensional dynamic risk assessment of network security. This paper will conduct research and analysis on the key technologies of network security multi-dimensional dynamic risk assessment.
\end{abstract}

\section{Introduction}

Nowadays, human beings have become dependent on the network. Relevant statistics show that there are as many as 700 million Internet users in China. People conduct online business negotiations, transaction payments, and resource sharing through the Internet. Since the birth of the network, security issues have received much attention from all walks of life. Some inconveniences in network applications may be attacked or invaded. Some hackers can use Trojans or backdoor software to steal their accounts, passwords, and online banking information, causing network users to suffer economic losses. The loss of personal information is relatively small, but if national information or corporate information is stolen, it will affect social harmony and stability. Corporate information is mostly trade secrets. Once leaked, it is very likely to affect normal operations, which will have a negative impact on the company and even induce the company to close down. In order to ensure network security and improve network security, it is of great significance to do a multi-dimensional dynamic risk assessment of network security ${ }^{[1]}$.

\section{Current status of network security multi-dimensional dynamic risk research and its significance}

Computer network refers to the sharing of information resources between multiple computers and their external devices with independent functions in different geographical locations through communication lines, under the management and coordination of operating systems and network software and hardware under network communication protocols. And the computer system that is passed. The purpose of network construction is to interconnect computers, share information, and integrate resources. The development of computer networks has been divided into four stages: remote terminal connection phase, computer network phase, network interconnection phase, Internet and information highway phase. The computer network is open and has a large user base, 
so anyone can become a network user. After entering the 21st century, the network has almost universalized promotion and application worldwide, and there are countless users in the global network. However, it is precisely because of the open nature of the network that it has also facilitated some unscrupulous people. The cyberattack not only has caused adverse effects on the society, but also brought economic losses to some enterprises ${ }^{[2]}$.

In recent years, cybersecurity has become a focus of widespread concern in the community. Many scholars have conducted research on the multidimensional dynamic risk of cybersecurity. Professor Lin Wen once proposed in the paper "Multi-level Dynamic Risk Quantitative Evaluation of Hierarchical Network Security" that network security is a prerequisite for network applications. If the security of the network cannot be guaranteed, the user's private information will be leaked, and the user will gradually move away from the network. This will undoubtedly restrict the development of the network. Nowadays, the network industry has become an economic pillar industry. If the network industry is retrogressed, it will inevitably have an adverse impact on economic development. The multi-dimensional dynamic risk of network security cannot be ignored. In recent years, cyber attacks have been written, network risks have increased, and multidimensional dynamic risks of network security have been evaluated. It is imperative to evade cyber risks scientifically.

\section{Common cyber attacks that threaten network security}

There are a lot of uncertainties and insecure factors on the network. Since the birth of the network, there have been acts of illegal intrusion, data theft and destruction. There is no $100 \%$ secure network in the world, and information theft, hacking, and virus attacks occur frequently in network development and applications. In 2015, Ctrip Network suffered a network attack, resulting in the inaccessibility of the website, the loss of user information, and the complete paralysis of the APP. The attack caused Ctrip to suffer direct economic losses of more than five million dollars, and the stock fell by 11.2 percent. There are many kinds of cyber attacks, but most of them are attacked by software vulnerabilities, physical vulnerabilities and data vulnerabilities, threatening network security, to achieve interception, stealing, cracking user information and destroying user systems. In order to conduct effective multi-dimensional dynamic risk assessment of network security, it is necessary to understand the means of network attacks. Common cyber attacks include IP spoofing attacks, password attacks, data hijacking attacks, and network eavesdropping attacks ${ }^{[3]}$.

Among them, password attack is one of the most common cyberattacks commonly used by hackers. After the hacker determines the target of the attack, he can use the exhaustive method to test the password and crack the network password through the dictionary. Password Attack In UNIX system cyber attacks, UNIX systems are vulnerable to password attacks because UNIX systems do not prompt or block user systems for incorrect password attempts. When the password test succeeds, the system will not send a report to the administrator when the network is compromised. The hacker can perform system data encryption file cracking through FTP or Telnet. Data hijacking attacks and network eavesdropping attacks in cyber attacks are extremely harmful, which will directly cause leakage of user password information, causing the network to fall into paralysis. Such attacks usually occur during network file transmission. The IP spoofing attack is a popular attack method. This attack mainly occurs. When the IP protocol transmits a message. Through analysis, it is not difficult to see the harm and seriousness of network attacks. Network attacks are ubiquitous. Network applications must be based on the characteristics and characteristics of network attacks, improve security risk assessment, improve network security, and reduce network risks ${ }^{[4]}$. The statistics of network security incidents are shown in figure 1. 


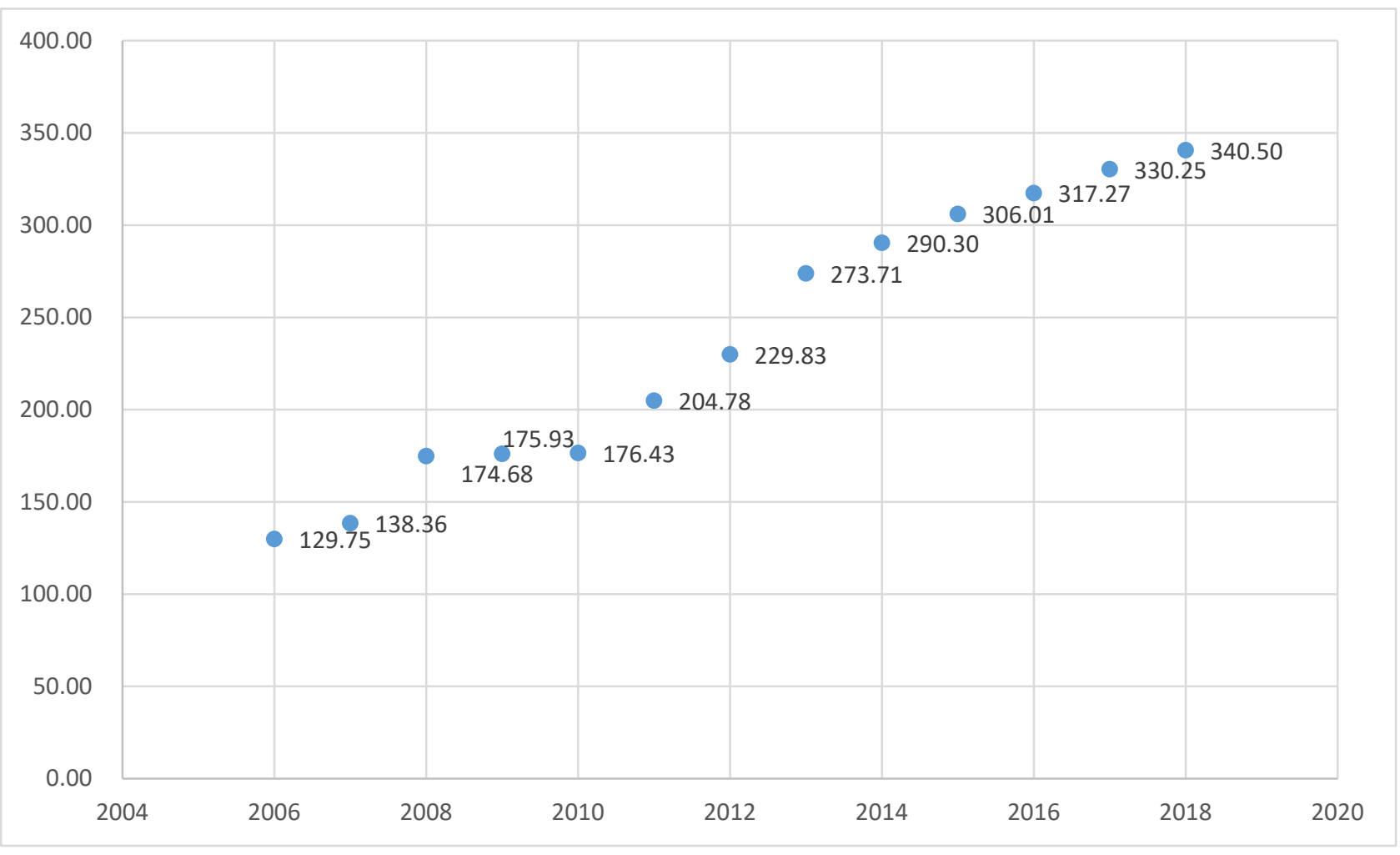

Figure 1: Statistics of network security incidents

\section{Key Technologies of Network Security Multidimensional Dynamic Risk Assessment}

Network security multi-dimensional dynamic risk threatens network user system security, information security, network security, evaluates network security multi-dimensional dynamic risk, and builds network risk assessment framework, which is a prerequisite for network security protection, and is important for improving network security. significance. Here are a few key points to analyze the key technologies of network security multi-dimensional dynamic risk assessment. The key technologies of network security multi-dimensional dynamic risk assessment are shown in the following figure 2 .

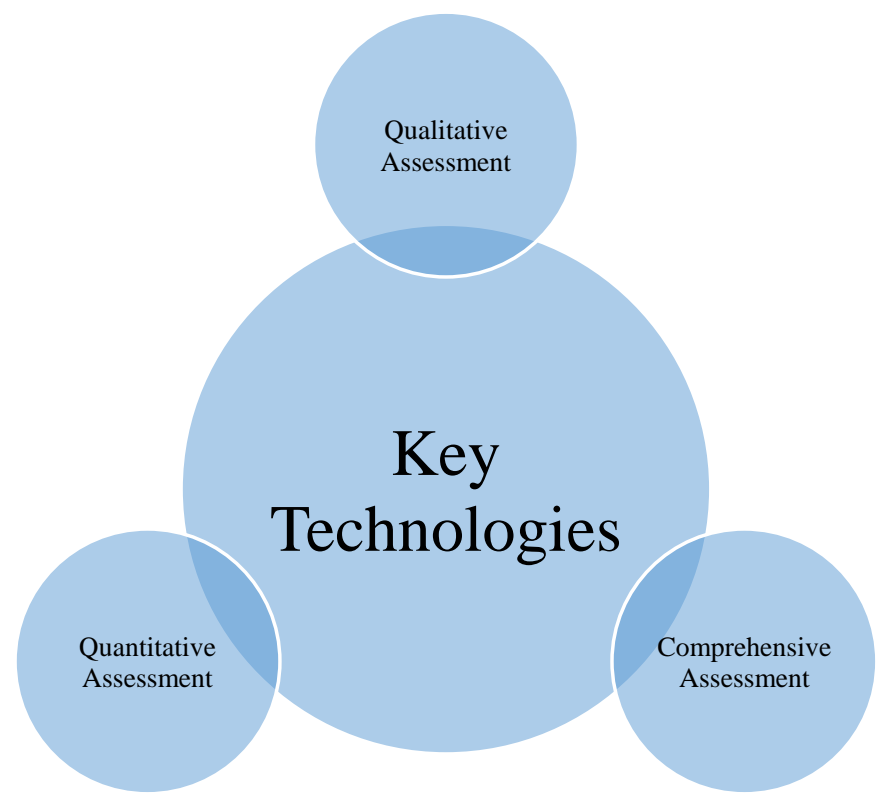

Figure 2: Key technologies of network security multi-dimensional dynamic risk assessment 


\subsection{Qualitative assessment techniques}

Qualitative assessment technology is a more commonly used multi-dimensional dynamic risk assessment technology for network security. The Delphi method is used to analyze the network security and judge the network security status by using the deductive deduction theory. In the specific evaluation process, the qualitative evaluation technology should first use the back-to-back communication method to obtain the security impact factors, use the anonymous data screening method to analyze the data processing results, and judge the network security multi-dimensional dynamic risk factors and network safety factors through multiple feedbacks and consultations, conducting a security risk assessment.

\subsection{Quantitative assessment techniques}

Compared with other evaluation techniques, the quantitative evaluation technology is more intuitive and the evaluation is more effective, but the evaluation complexity and difficulty are limited, and there are certain limitations in the application. This kind of evaluation technology mainly uses the weighted coefficient method to conduct multi-dimensional dynamic risk assessment of network security through data index quantification. The principle of quantitative evaluation technology is to use the weight coefficient method to calculate the parameter weight, measure the system uncertainty, quantify the security risk, and evaluate the network security multi-dimensional dynamic risk according to the extreme value feature. If the entropy value is larger in the assessment, the multi-dimensional dynamic risk of network security is larger, and the more influence factors of security risk. If the maximum value of the entropy value is 1 , the smaller the risk factor has on the system security, the higher the network security.

\subsection{Comprehensive assessment technology}

The multi-dimensional dynamic risk of network security has many influencing factors, such as variability and complexity. In some cases, if it is impossible to obtain good evaluation results through qualitative assessment techniques or quantitative assessment techniques, comprehensive assessment techniques can be applied. The comprehensive evaluation technology is to improve the effectiveness and accuracy of the evaluation by combining various evaluation techniques, to judge the network safety factor, and to achieve the purpose of multi-dimensional dynamic risk assessment of network security. The comprehensive evaluation methods mainly include: threat tree method, obstacle tree method, and analytic hierarchy process. Most of the comprehensive evaluation techniques are based on quantitative evaluation, with qualitative assessment as the core, inheriting the advantages of these two evaluation techniques, and further improving the evaluation accuracy ${ }^{[5]}$.

\section{Conclusion}

Security is the foundation and prerequisite for network applications. It is essential to ensure network security. The multi-dimensional dynamic risk of network security threatens the harmonious construction of network society and the security of network information data. It is of great significance to strengthen the application and promotion of multi-dimensional dynamic risk assessment technology for network security.

\section{References}

[1] Mashor Housh,Ziv Ohar. Integrating physically based simulators with Event Detection Systems: Multi-site detection approach[J]. Water Research,2017,110.

[2] Christopher W. Badenhop,Scott R. Graham,Benjamin W. Ramsey,Barry E. Mullins,Logan O. Mailloux. The Z-Wave routing protocol and its security implications[J]. Computers \&amp; Security,2017,68.

[3] Rajbir Kaur,Navroop Kaur,Sandeep K. Sood. Security in IoT network based on stochastic game net model[J]. International Journal of Network Management,2017,27(4). 
[4] Angsheng Li,Xiaohui Zhang,Yicheng Pan. Resistance maximization principle for defending networks against virus attack[J]. Physica A: Statistical Mechanics and its Applications,2017,466.

[5] Mashor Housh,Ziv Ohar. Integrating physically based simulators with Event Detection Systems: Multi-site detection approach[J]. Water Research,2017,110. 